\title{
AUSENCIA DE DIRECCIÓN Y LIDERAZGO Y CRECIMIENTO DE ANOMIA EN ENTIDADES DEL SECTOR PÚBLICO EN EL PERÚ
}

\section{LACK OF DIRECTION AND LEADERSHIP AND ANOMIE GROWTH IN THE PUBLIC-SECTOR ENTITIES IN PERU}

\author{
Alberto Benjamín Espinoza Valenzuela \\ Doctor en Ciencias Contables y Empresariales y Magíster en Administración - Universidad Nacional Mayor de San Marcos \\ Email: aespinozaoci@gmail.com (Autor Corresponsal)
}

\section{[Recibido: 17/05/2016 Aceptado: 24/08/2017]}

\section{RESUMEN}

El Estado como toda organización necesita adoptar modelos de Dirección y Gerencia que aseguren los fines y objetivos del buen uso de los presupuestos públicos que se le asignan. Al respecto en los últimos años se ha evidenciado dos fenómenos que han imposibilitado este manejo. Por un lado, la ausencia de funcionarios con capacidad para sumir la Dirección y Gerencia en las Entidades Estatales en el Perú, y por otro, lado el accionar "punitivo" del servidor público caracterizado por la "anomia" sobre la que basa sus actos, el mismo que muchas veces es inculcado al interior del estado, por prácticas y accionar nada eficientes, eficaces y transparentes, sino adquiridos en su formación socio-cultural como es la "anomia moral" caracterizada por la llamada "Tolerancia media". Específicamente a través del presente trabajo se analizan y describen las características y tendencias del accionar del trabajador estatal ante estos dos fenómenos cuasi estructurales. Además de las consecuencias que esta situación genera en sus relaciones con el resto de la economía, caracterizada por altos niveles de corrupción y una inadecuada prestación de los servicios públicos para los cuales han sido creados, garantizando la efectividad del gasto en el marco normativo que la legalidad exige para el efecto.

\section{PALABRAS CLAVE}

Dirección, liderazgo, anomia, corrupción

\begin{abstract}
The State, like any organization, needs to adopt leadership and management models that ensure the purposes and objectives of the good use of the assigned public budgets. In this regard, in recent years, two phenomena have made this management impossible. On the one hand, the absence of officers with the capacity to take over the Direction and Management in the State Entities in Peru, and on the other hand, the "punitive" action of the public servant characterized by the "anomie" on which his acts are based, the same that is often inculcated within the state, by not efficient practices and actions, effective and transparent, but acquired in their socio-cultural formation as is the "moral anomie" characterized by the so-called "Medium tolerance". Specifically, through the present work, the characteristics and tendencies of the state worker's actions are analyzed and described before these two quasi-structural phenomena. In addition to the consequences that this situation generates in relation to the rest of the economy, characterized by high levels of corruption and inadequate provision of public services for which they have been created, guaranteeing the effectiveness of the expenditure in the regulatory framework that the legality demands for that effect.
\end{abstract}

\section{KEYWORDS}

Direction, leadership, anomie, corruption. 


\section{INTRODUCCIÓN}

En los últimos años y de acuerdo a los casos de corrupción, hablar de transparencia pública se ha convertido en una necesidad de extrema trascendencia. Y en una Sociedad como la nuestra en que la ética es una variable que no se aprecia con frecuencia en la administración pública, es necesario reflexionar sobre los niveles de gerencia que debe garantizar el buen gasto estatal, los mismos que deben estar caracterizados por niveles idóneos de dirección y liderazgo.

Y es que el estudio de dirección y liderazgo en el sector público debe asegurar total transparencia en el uso de los recursos públicos, conceptualizando una forma innovadora de esquematizar la gestión en la organización estatal suponiendo adoptar nuevas formas de dirigir; situando al Titular del Pliego (ministro, alcalde, presidente regional, jefe etc. ) y a su entorno, en gestores que determinen esquemas de éxito de dirección y sobre todo la transparencia que buena falta le hace a la mayoría de las organizaciones públicas en el país.

Particularmente y al igual que el Dr. Bravo (2009) refiere que debemos trascender las teorías básicas de la administración y sus diversos modelos aplicativos. En este sentido, el presente estudio se sitúa en una posición de análisis a la problemática de la gerencia en la organización pública por parte del "Director" (Liderazgo), "Colaboradores", funcionarios y trabajadores públicos con niveles de "anomia moral y social", en base a lo cual de acuerdo a los niveles elevados de corrupción, proponen diversas interrogantes, que nos plantean objetivos e hipótesis de acción para proyectar las soluciones a que hubiera lugar.
La gerencia pública como instrumento de gobierno y desarrollo social

Tomando una frase de Covey (2003) "El líder del futuro, del próximo milenio será el que cree una cultura o un sistema de valores centrados en los principios" es necesario considerar las características del gerente público, conforme a las diferencias que existen en asumir compromisos de Dirección en el ámbito privado a diferencia del sector público. Al respecto no se ha analizado quizá como corresponde el concepto y papel que debe caracterizar la actuación del funcionario público a quien se le encarga administrar los recursos del estado en un marco de objetivos y metas sociales a cumplir.

\section{Al respecto surgen algunas interrogan- tes}

¿La administración pública es un modelo de administración moderna o es un modo técnico de gerenciar, que empíricamente afronta el trabajo de dirección o gerencia en los organismos estatales y que a la postre resulta en su aplicación un mecanismo incipiente de dirección que no logra los objetivos deseados?

Si llevamos esta interrogante a la totalidad de trabajadores de una institución estatal ¿Podemos asegurar niveles adecuados de integridad $y$ transparencia en el manejo de los recursos públicos?

Partiendo de estas interrogantes, es conveniente proponer un primer nivel de análisis, sobre la base de una de las fuentes de estudios innovadoras de los últimos tiempos propuesta por Peter Drucker a través de la obra escrita por Hass Edersheim (2007), el cual señala definiciones precisas sobre el concepto de "gerencia" que pue- de servir a los objetivos de analizar su alcance en las instituciones públicas. $\mathrm{Al}$ respecto se precisa que gerenciar es ser eficiente y efectivo; ser eficiente es hacer las cosas correctamente, en un clima de transparencia mientras que la efectividad es hacer las cosas correctas en el marco de los objetivos establecidos. Hacer las cosas correctamente en el sector público es un principio básico de Dirección y Gerencia que se busca permanentemente en el manejo de la cosa pública a fin de cautelar, preservándolos intereses del estado.

Sin embargo, se observa permanentemente como este principio "horizontal" de gestión se rompe al descartar políticas públicas de corto, mediano y largo plazo que elude la PLANIFICACIÓN como primer requisito indispensable de una adecuada gerencia. Vemos con poco agrado como cada vez que un nuevo titular de pliego al asumir sus funciones requieren $y$ exigen de su personal cambios poco menos que estructurales pero poco sustanciales en la organización, (nueva estructura organizacional), Nuevo Manual de Organización (MOF) nuevo Reglamento de Organización de Funciones (ROF), con el ánimo de realizar cambios diferentes en la organización y hacer sentir el cambio de dirección, amén del rompimiento de políticas públicas que de alguna manera han podido acercar el gasto a las necesidades de la población o usuarios del servicio público al que representan. En este sentido, se rompe un principio de administración moderna en el cual la ORGANIZACIÖN debe ser el resultado de premisas básicas de "visión y misión" institucional y políticas públicas de gestión estratégica estructural, y no obedecer a cambios políticos solo de trascendencia coyuntural de trascendencia cortoplacista. 
Es necesario remarcar que el Estado peruano cuenta con un sistema de planificación formal representado por CEPLAN, pero que sin embargo el país no cuenta con un Plan de Desarrollo Nacional que perdure en el tiempo y que resuelva los problemas nacionales partiendo de un conjunto de políticas públicas que trasciendan a gobiernos de turno. Esto finalmente nos conduce a una ejecución presupuestal sin un marco articulado de desarrollo con las diferentes instituciones no sostenibles en el tiempo.

Y si no existe un horizonte claro de políticas públicas en la Institución no habrá políticas claras de dirección o gerencia. Las marchas y contramarchas de dirección en las organizaciones públicas se pueden apreciar en los cambios de Titulares de Pliego en el gobierno central, región o local.

Es decir no se plantean modelos que bajo conceptos teóricos o conceptuales busquen coherencia y continuidad en los métodos de dirección y gerencia en todos los niveles de la organización. Si bien es cierto la eficiencia y la efectividad no pueden esperar en el logro de resultados e impacto en los servicios público que brindan las instituciones estatales, hasta ahora no se aprecian cambios sustanciales en el "manejo de los recursos públicos" que asocien dirección, transparencia y efectividad en su uso, que debe ser relativamente sencillo más aún cuando el funcionario y servidor públicos prácticamente tienen diseñados los procedimientos del compromiso presupuestal, devengado y pagado y solo pueden cumplir con sus labores en el marco de las normas legales que enmarcan su accionar.

$\mathrm{Al}$ respecto si tomamos entre otros autores a Igor (1998) quien manifiesta:

“...La dirección estratégica es un enfoque sistemático hacia una responsabilidad mayor y cada vez más importante de la gerencia general, es decir relacionar a la empresa con su entorno, estableciendo su posición, de tal manera que garantice su éxito continuo y la proteja de las sorpresas".

Y Drucker (1982) que señala que:

"Una gestión exitosa depende de la capacidad de su gente, del adecuado trabajo de grupo, del conjunto de los seres humanos, de la participación para solucionar no solamente los problemas sino los retos del marketing permanente $y$ del proceso de inmersión y globalización de los mercados. El éxito organizacional se mide por la capacidad de su gente, por el conocimiento de los mismos...."

Asimismo precisa: “... El valor que el cliente le da al producto que se ofrece está directamente relacionado con el uso que le da y la satisfacción del mismo para con sus necesidades. Es sobre esta perspectiva que se debe trabajar a costas del conocimiento cada vez más complejo para satisfacer de las mejores formas, esas necesidades...”.

Las premisas anteriormente señaladas como ejes centrales para una buena gerencia pública no han sido consideradas en su aplicación. Las instituciones públicas anteriormente y en la actualidad vienen siendo dirigidas sin "rumbo" administrativo gerencial, caracterizado por una relación empírica con la realidad en la cual se privilegia el gasto, careciendo del carácter predictivo que requiere la organización interna de la entidad que la haga más eficiente, eficaz, en mérito a las necesidades de organización con transparencia interna, un compromiso serio y real con el cliente externo que es la población, para cuyo servicio son específicamente nombrados.

El estudio es de carácter descriptivo a nivel teórico, de diseño no experimental con un enfoque cualitativo, focalizado con tratamiento de análisis documental con técnicas descriptivas de su contenido, revisándolas y analizándolas para comprender la realidad de los niveles de dirección y liderazgo en la administración pública y como su ausencia viene generando anomia, por ende corrupción en el país.

El estudio al no ser cuantitativo, no presenta necesariamente universo y población por cuanto el análisis es de contenido, se recoge información especializada sobre la materia, a través de fichas, textos, opiniones, resúmenes etc.

Sin embargo, a pesar de la presencia de SERVIR para profesionalizar el Estado con la concurrencia de profesionales calificados en los principales puestos directivos de acuerdo a estadísticas de PROETICA, en su encuesta al 2015, señala como resultado que el nivel de la corrupción se ha incrementado en los últimos 6 años, pasando de un $49 \%$ a un $61 \%$ de corrupción entre funcionarios y autoridades.

Igualmente, en los últimos 6 años, si bien la percepción de ineficiencia de funcionarios $y$ autoridades ha pasado de 25 a $14 \%$, aún sigue elevada el índice de percepción en un 75\% para ambos conceptos. 


\section{PRINCIPALES PROBLEMAS DEL ESTADO PERUANO}

La corrupción de funcionarios u autoridades es, por amplio margen, el principal problema

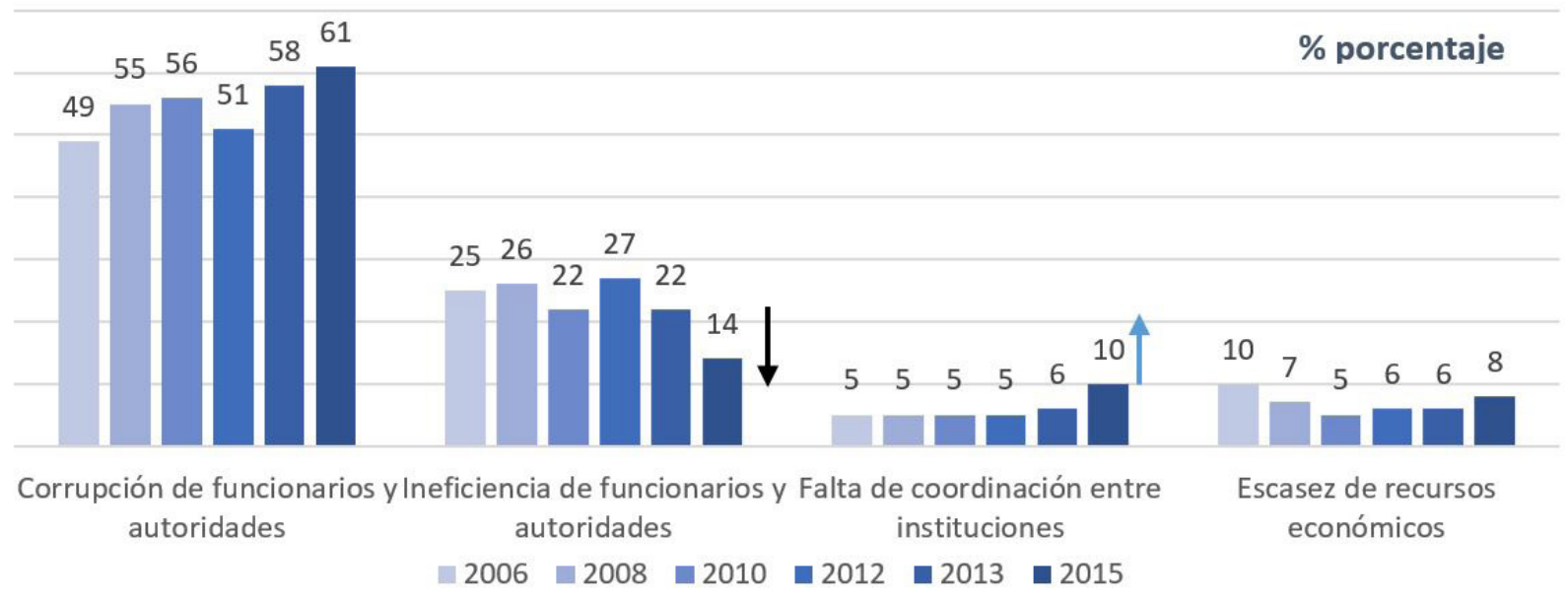

Figura 1. Principales problemas del Estado peruano

Fuente: Proética

Esta situación sin lugar a dudas tiene un componente político, los principales cargos gerenciales son otorgados a personas vinculadas políticamente al partido de gobierno o amigos cercanos.

¿Es posible que esta situación a pesar de los esfuerzos que hace el estado a través de SERVIR, se deba en gran medida a la falta de liderazgo y dirección por parte de las principales autoridades del país y a la ausencia de un estilo de gerencia público que luche frontalmente contra la corrupción a través de mejores alternativas de gestión?

La práctica de un estilo propio de dirección y liderazgo no debe ser aislada del resto de la organización debido principalmente al carácter público de sus funciones. Los empleados quieren saber cuál será su situación, tanto en términos básicos de estabilidad y se- guridad laboral en el futuro como en el campo de desarrollo profesional y personal sobre todo la política de transparencia en manejo de los recursos públicos que se implementará por parte del nuevo titular de pliego así como los funcionarios que se convoquen.

Es usual escuchar en las organizaciones estatales conceptos sobre principios de dirección, gerencia y responsabilidad, y hasta de transparencia pero los niveles de coordinación real sobre estos aspectos no se perciben claramente en las relaciones laborales que se tiene con la llamada "Alta dirección". Escuchamos mucho sobre conferir poder a los empleados y sobre la diversidad en las organizaciones. ¿Cómo es que estas dos situaciones afectan el lugar de trabajo?

La diversidad es importante. Y la responsabilidad también. La atención se debe centrar en encontrar las maneras de integrar las perspectivas y talentos de personas de todas las procedencias y su sentido de responder ante los diversos niveles de corrupción que pueden amenazar a la entidad. Cuando todos son valorados y capaces de contribuir con su talento, el Estado gana, el compromiso se incrementa y la transparencia se impone. Este debe ser el primer paso para evitar y disminuir la "anomia" que pone en riesgo el uso adecuado de los presupuestos asignados a la entidad.

En este sentido es de suma importancia para la efectividad de la dirección y liderazgo que la entidad pública aplique una política de Recursos humanos adecuada a la cultura laboral de la organización. En el siguiente cuadro se puede apreciar una muy mala percepción de las instituciones en la lucha contra la corrupción. 
PERCEPCIÓN Y EVALUACIÓN DE INSTITUCIONES

Exceptuando por los Medios de Comunicación y la Defensoría, la mayoría califica negativamente el

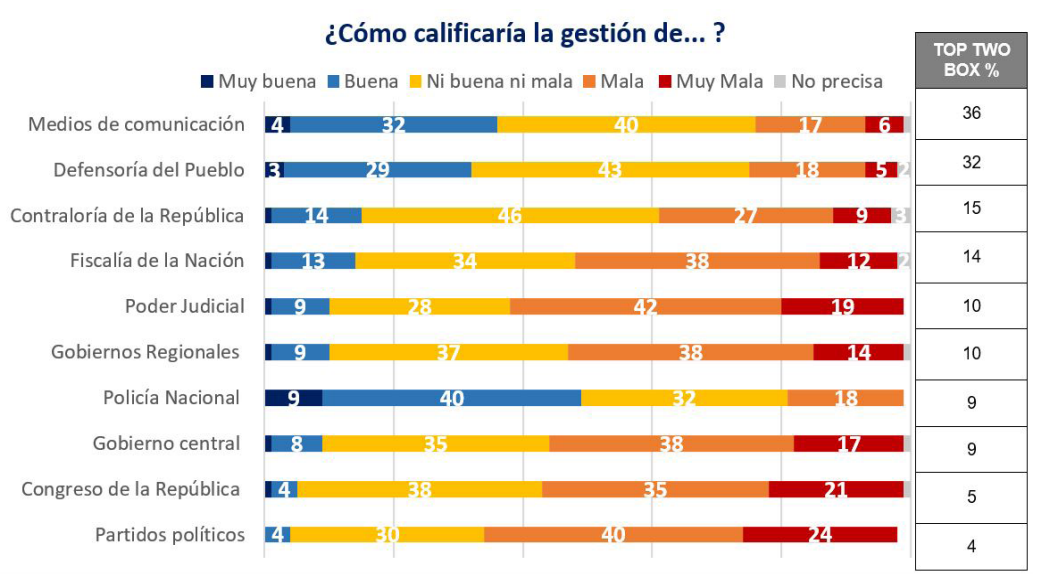

Figura 2. Percepción y evaluación de instituciones / Fuente: Proética

Tubino (2004) profesor y filósofo universitario, señala, a partir del concepto acuñado por La teoría del desarrollo cognitivo, teoría planteada por Jean Piaget. Del cual, su discípulo Lawrence Kohlberg profundizó en los estudios acerca del desarrollo cognitivo, señala las causa por las que en nuestro contexto, la moral convencional ha devenido en moral preconvencional, planteando que la recuperación de la ética pública pasa necesariamente por la formación de una cultura de la ciudadanía que haga posible la subordinación de los valores privados a los valores públicos.

Concluye en su razonamiento lógico:
“... ¿De qué sirven los recursos públicos si son mal empleados? O lo que es más grave aún, ¿De qué sirven los recursos públicos cuando, por actos sistemáticos de corrupción, son desviados para fines privados?...Se refiere a la corrupción institucionalizada, tolerada por la gente y por ello mismo crónica”.

Definitivamente nos encontramos en un estadio de anomia moral y social en la cual la ciudadanía acepta niveles de corrupción como prácticas usuales de obtener dinero y lograr objetivos personales. ¿Dónde termina el negocio y empieza la corrupción? En este sentido, el nivel de tolerancia media a la corrupción empieza a definir niveles de aceptación en las cuales vemos lo anormal como normal y viceversa, y nos acostumbramos a ver en nuestras autoridades niveles aceptables de corrupción mientras "hagan obras".

El tratamiento de la corrupción, debe ser visto a través de la actuación de los agentes económicos de toda sociedad, familias, empresas y estado. Si bien los valores éticos y morales deben ser vistos estructuralmente en los primeros años del individuo, conculcados al interior de las familias, estos deben consolidarse en el plano empresarial y gubernamental con prácticas adecuadas de solvencia moral y ética.

\section{PERCEPCIÓN DE CORRUPCIÓN EN INSTITUCIONES}

Mientras la percepción de corrupción en el Congreso y la PNP disminuye, se incrementa la de los partidos políticos. Lima suele ser más crítica que el interior salvo en el caso de los gobiernos regionales.

$$
\text { ¿Qué instituciones que conoce están entre las tres más corruptas? }
$$

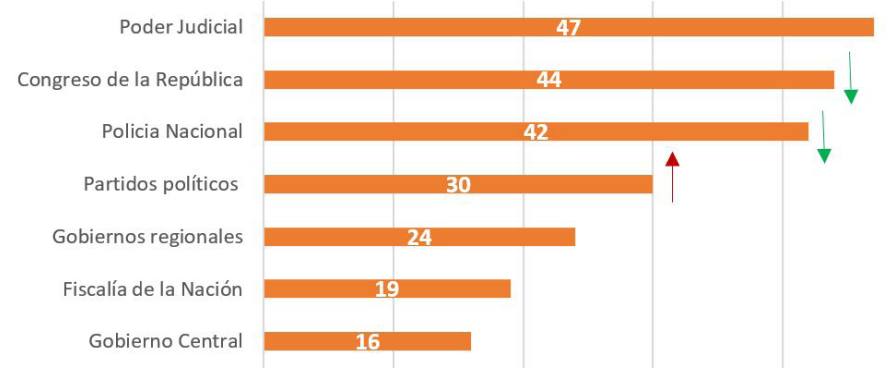

\begin{tabular}{|c|c|}
\hline 2013 & 2012 \\
\hline 49 & 56 \\
\hline 55 & 47 \\
\hline 53 & 52 \\
\hline 22 & 27 \\
\hline 24 & 20 \\
\hline 13 & 16 \\
\hline 11 & 9 \\
\hline
\end{tabular}

\begin{tabular}{|c|c|}
\hline Lima & Interior \\
\hline 52 & 43 \\
\hline 50 & 40 \\
\hline 41 & 43 \\
\hline 34 & 27 \\
\hline 18 & 28 \\
\hline 21 & 18 \\
\hline 16 & 15 \\
\hline
\end{tabular}

Figura 3. Percepción de corrupción en instituciones / Fuente: Proética 
Pero justamente ocurre lo contrario a nivel de empresas y gobierno. $Y$ es justamente el "Estado" quien debe iniciar y mantener una lucha frontal contra la corrupción, quien la inicia, mantiene y esconde a través de la impunidad. La percepción de la ciudadanía responde a este fenómeno a través de los resultados de la encuesta mostrada.

Si observamos que entre las entidades más corruptas se encuentran El Poder
Judicial, Congreso de la República y Policías Nacional, los niveles de respeto a la sociedad y normas convencionales que priman en las relaciones interpersonales por parte de la población se ven absolutamente resquebrajadas.

En el siguiente cuadro se aprecian resultados de la percepción de los encuestados sobre cómo el gobierno central enfrenta los actos de corrup- ción. Si vemos que la entidades del gobierno central principalmente son los responsables de administrar adecuadamente los recursos públicos del país, no existe una administración que con niveles adecuados de dirección y liderazgo asuman este papel.

Si sumamos los niveles de aceptación de la corrupción en una lucha poco frontal entre poco eficaz y nada eficaz, el resultado es alarmante en un $85 \%$.

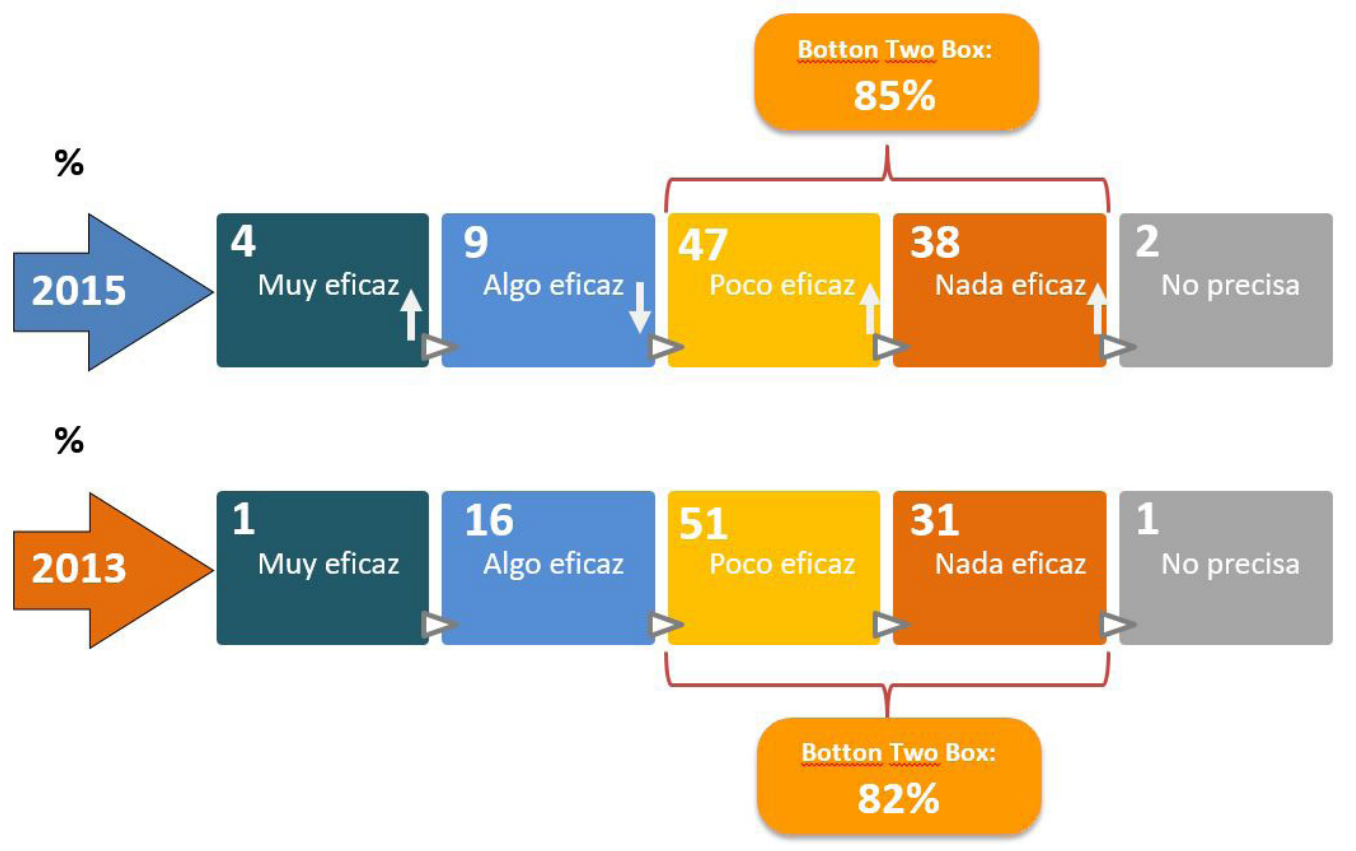

Figura 4. Eficacia en el combate de la corrupción / Fuente: Proética

Es por ello que surge la primera interrogante sobre el papel del director y funcionarios públicos ante los niveles de anomia y alta corrupción en el país ¿Cómo entender y aplicar la gerencia pública en un entorno variado y distinto en gerentes y trabajadores de distinta formación, personalidad, cualidades, experiencia, etc.?

La respuesta no puede ser sencilla puesto que la percepción de los trabajadores y funcionarios al interior de la entidad pública los sitúan permanentemente en posiciones distintas y hasta antagónicas en el límite de sus propias funciones. El titular de pliego, directivo y funcionarios, en este sentido tienen que ser no solamente un administrador que lleve la ges- tión a las intenciones personales de los trabajadores sino que consiga los resultados y las metas esperadas por la institución. Caso contrario y como consecuencia de esa falta de probidad institucionalizada a falta de dirección y liderazgo se corre el riesgo de caer en la llamada "Tolerancia media a la corrupción”. 


\begin{tabular}{|c|c|c|c|c|c|c|c|c|c|c|c|c|c|c|c|c|c|c|}
\hline \multirow{4}{*}{$\begin{array}{l}\text { Que un funcionario público } \\
\text { favorezca a parientes y amigos }\end{array}$} & \multirow{2}{*}{\multicolumn{6}{|c|}{$\begin{array}{l}\text { ALTA TOLERANCIA } \\
\text { \% Totalmente de acuerdo } \\
\text { +\% De acuerdo }\end{array}$}} & \multicolumn{6}{|c|}{ ALTA TOLERANCIA } & \multicolumn{6}{|c|}{ RECHAZO DEFINIDO } \\
\hline & & & & & & & \multicolumn{6}{|c|}{$\begin{array}{l}\% \mathrm{Ni} \text { de acuerdo ni en desacuerdo } \\
+\% \text { En desacuerdo }\end{array}$} & \multicolumn{6}{|c|}{$\%$ Totalmente en desacuerdo } \\
\hline & 2015 & 2013 & 2012 & 2010 & 2008 & 2006 & 2015 & 2013 & 2012 & 2010 & 2008 & 2006 & 2015 & 2013 & 2012 & 2010 & 2008 & 2006 \\
\hline & 8 & 12 & 7 & 12 & 6 & 7 & 68 & 71 & 65 & 69 & 72 & 65 & $23 \uparrow$ & 17 & 27 & 18 & 21 & 27 \\
\hline $\begin{array}{l}\text { Dar un obsequio o dinero para } \\
\text { agilizar un trámite público }\end{array}$ & 11 & 10 & 4 & * & $*$ & * & 71 & 73 & 65 & $*$ & * & $*$ & 20 & 17 & 31 & * & $*$ & $*$ \\
\hline $\begin{array}{l}\text { Pagar "propina" para que le } \\
\text { perdonen una multa }\end{array}$ & 4 & 8 & 2 & 4 & 3 & 5 & 774 & 73 & 64 & 76 & 78 & 67 & 18 & 19 & 34 & 20 & 18 & 27 \\
\hline $\begin{array}{l}\text { Llenar documentos con datos } \\
\text { falsos por conveniencia }\end{array}$ & 3 & 4 & 0 & 1 & 0 & 0 & $74 \uparrow$ & 69 & 64 & 76 & 74 & 65 & $22 \nabla$ & 26 & 36 & 23 & 25 & 34 \\
\hline $\begin{array}{l}\text { Robar servicios públicos (agua, } \\
\text { luz, etc) }\end{array}$ & 2 & 4 & 0 & * & * & $*$ & $72 \uparrow$ & 66 & 62 & $*$ & * & $*$ & $25 \downarrow$ & 29 & 38 & $*$ & $*$ & $*$ \\
\hline Promedio & 6 & 8 & 3 & 6 & 3 & 4 & 72 & 70 & 64 & 74 & 75 & 66 & 22 & 22 & 33 & 20 & 21 & 29 \\
\hline Base: Total de entrevistados (13 & & & & & & & & & & & & & & & & & & \\
\hline
\end{tabular}

Figura 5. Tolerancia hacia la corrupción / Fuente: Proética

Se aprecia por ello que la administración pública en manos del funcionario, directivo y trabajador públicos todavía se encuentran en evidente etapa evolutiva, que aún no han encontrado un derrotero (camino) que le permita transcender las teorías básicas de la administración y sus diversos modelos aplicativos, buscando nuevas proposiciones hipotéticas que le permitan derivar en nuevas y útiles herramientas de aplicación teóricas y técnicas al interior de las entidades públicas para administrar correctamente los presupuestos del estado. Más aún cuando el 78\% de la población considera tolerable la corrupción en situaciones que van desde robar servicios públicos, llenar documentos falsos, pagar coimas o que un funcionario favorezca a parientes y amigos.

\section{CONCLUSIONES}

1. Público el titular de pliego, director y funcionario público debe ser ejercido por una persona con característica de líder que asegure el éxito de la gestión, principalmente en la aplicación de la estrategia, la visión y la misión, como indicadores naturales de la ruta a seguir. Evitándose el nombramiento por factores políticos o de confianza en personas que no cuenten con el perfil requerido.

2. El primer objetivo del director público es lograr la difusión del conocimiento y comportamiento ético en el personal que tiene a su cargo los diversos objetivos que enlazan los fines y metas de la empresa interiorizados en cada una de las unidades orgánicas que la componen. Haciendo productivo el conocimiento que tiene cada una de las personas como parte de su formación individual debidamente concordado con los objetivos de la organización para el logro final de la visión que se ha proyectado conjuntamente con niveles adecuados de transparencia en el manejo de los recursos públicos.

3. El director público debe tener claramente entendida la visión de la empresa en materia de transparencia respecto al uso de los presupuestos públicos y proponerla como mecanismo de acción a la voluntad de todos los trabajadores, mediante estrategias adecuadamente diseñadas al interior de la entidad pública que evite malos manejos y actos de corrupción.

4. Por ahora dirección y liderazgo en el sector público es solo una forma empírica de gestionar que no garantiza el uso apropiado de los recursos públicos y por ende pone en duda su efectividad conceptual, que condiciona su aplicación a las variables endógenas ( Recursos Humanos ) y exógenas ( políticas ) a las que se ve expuesta.

\section{REFERENCIAS BIBLIOGRÁFICAS}

Bravo, J. (2009). Planificación Sistémica. Santiago de Chile: El Mercurio Aguilar.

Covey, R. (2003). Los 7 hábitos de la 
gente altamente efectiva: la revolución ética en la vicia cotidiana y en la empresa. Buenos Aires: Paidós.

Drucker, P.F. (1982). La Administración en una época de grandes cambios. Buenos Aires: Editorial Sudamericana.

Hass, E . (2007). Enseñanzas de Peter Drucker. USA: Mc Grawhill.
Igor H.A. (1998). La Dirección estratégica en la práctica empresarial. México DF: Ediciones Eddison Wesley Longman

Proética (2015). IX Novena encuesta nacional sobre corrupción 2015. Recuperado de https://es.scribd.com/ document/292794637/Novena-Encuesta-nacional-sobre-percepciones-de-la-corrupcion-2015
Tubino, F. (2004). Ética en la Vida Pública. Ponencia. Ética de Función Pública y Desarrollo de la Ciudadanía. Madrid: OEI. Universidad Antonio Ruiz de Montoya. 